\title{
COMMUNICATIONS
}

\section{EFFECT OF DIENCEPHALIC STIMULATION UPON INTRA-OCULAR PRESSURE*}

\author{
BY \\ J. GLOSTER AND D. P. GREAVES \\ Institute of Ophthalmology, \\ University of London \\ Director of Research: Sir Stewart Duke-Elder
}

THERE is much clinical evidence to suggest that certain types of glaucoma are related to activity of the autonomic nervous system. This does not imply that the aetiology of glaucoma is necessarily bound up with autonomic dysfunction, nor deny the importance of factors such as anatomical closure of the angle or increased resistance to outflow of aqueous humour as a result of changes in the trabecular network or beyond. The acute attack of glaucoma following an emotional disturbance is perhaps the most frequently encountered example of this suggested relationship.

Whilst much work has been done on the chemistry of the aqueous humour and its outflow, relatively little has been done on the nervous mechanisms influencing intra-ocular pressure. With regard to the latter, attention has been directed mainly to the peripheral nervous system. It is known, for example, that stimulation of the cervical sympathetic trunk and fifth cranial nerves in cats and rabbits is accompanied by changes in intra-ocular pressure, whilst stimulation of the third and seventh cranial nerves affects the intraocular pressure only in so far as the muscles supplied by these nerves contract and press on the eye causing a temporary acute rise in pressure (Greaves and Perkins, 1952, 1953, 1956; Perkins, 1955, 1957).

In view of the known effects of stimulation of the peripheral nerves and the probable influence of autonomic factors on intra-ocular pressure, it has been suggested that higher vegetative centres may exert some control upon the pressure within the eye, and recent evidence supporting this view has been clearly summarized by Sir Stewart Duke-Elder in the 1957 Bowman Lecture.

Undoubtedly, further physiological investigation is necessary and only in the last few years have specific attempts been made to investigate changes in intra-ocular pressure resulting from stimulation of the central nervous system; in particular, the diencephalon has received most attention, as this part of the brain is known to influence many bodily functions. Such studies have been reported by Schmerl and Steinberg (1950), Nagai and others (1951), Weinstein (1954), von Sallmann and Lowenstein (1955), and Gloster 
and Greaves (1956). In the last paper we presented a preliminary report of our results which are now dealt with in greater detail.

\section{Methods}

Stereotaxic Apparatus.-The principles of stereotaxic technique are well known, but to satisfy the special requirements of our experiments, the apparatus was designed to give good access to the eyes of the animal and to provide fixation of the inferior orbital margin without pressure on the eye-ball.

The apparatus is illustrated in Figs $1(a)$ and $1(b)$; it is seen to consist of a heavy base-plate $(B)$ with two vertical pillars $(P, P)$ carrying adjustable ear-plugs $(E, E)$; also a raised movable platform (D) with adjustable lugs to fix on the inferior orbital margins $(\mathrm{M})$ and hard palate $(\mathrm{N})$.

The electrode $(C)$ is carried in a chuck and collet held in an arm (S) attached to a modified compound lathe-slide (L). The compound slide allows movement in two directions at right angles in a horizontal plane. The electrode can be moved vertically, movement in this direction being measured by a millimetre scale on the electrode chuck.

The whole arrangement allows free movement of the electrode, and the provision of millimetre scales enables the position of the electrode tip to be determined with reference to three planes at right angles to one another:

(1) a horizontal plane passing through the central points of the ear-plugs and the points of contact of the lugs $(M)$ with the inferior orbital margins;

(2) a vertical plane passing through the centres of the ear-plugs;

(3) a vertical plane at right angles to (2) passing through the mid-line of the apparatus.

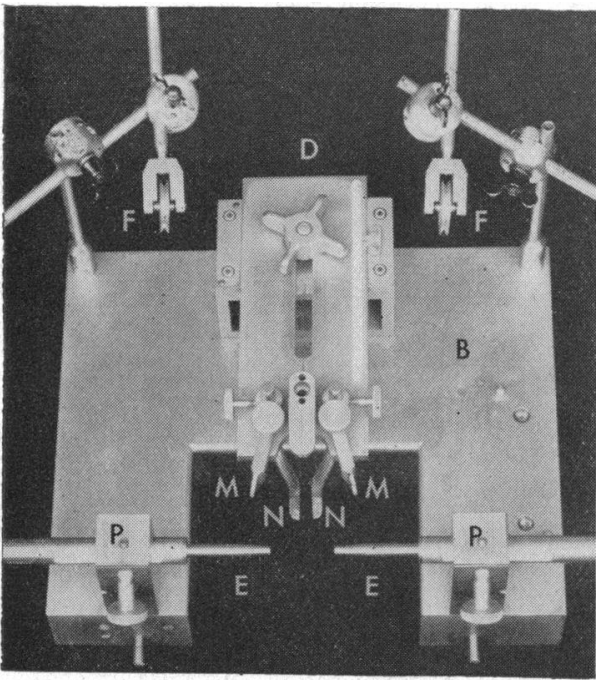

FIG. 1 (a).-Stereotaxic apparatus with electrode-carrier removed. For explanation of symbols see text.

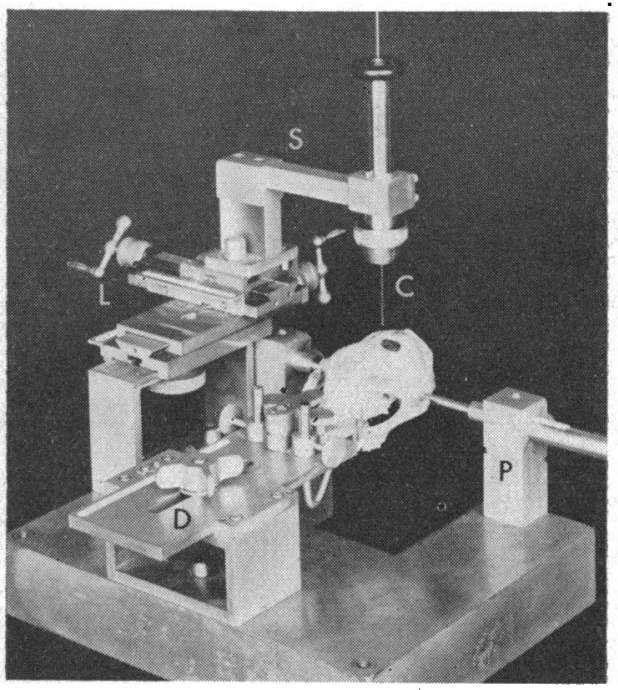

FIG. 1 (b).-Stereotaxic apparatus with electrode in carrier, skull positioned but nictitating membrane pulleys and their supports removed. For explanation of symbols see text. 
It is pertinent at this stage to emphasize our nomenclature. All frontal planes are defined in millimetres in front of the vertical inter-aural plane, prefixed by the letter "A", thus A12, A9, etc. Measurements in a vertical direction were referred to the horizontal plane passing through the external auditory meatuses and the inferior orbital margins; we termed this the "zero horizontal" or " $H$ " plane. Measurements to the right or left of the mid-line are given in millimetres prefixed by the letter " $R$ " or " $L$ " respectively. Thus a point in the brain referred to as "A12, H + 7, R3" is $12 \mathrm{~m}$. in front of the inter-aural plane, $7 \mathrm{~m}$. above the horizontal plane, and $3 \mathrm{~m}$. to the right of the mid-line.

Preparation of Animal.-Male cats weighing approximately $3 \mathrm{~kg}$. were anaesthetized by intravenous injection of a 1 per cent. solution of chloralose $(100 \mathrm{mg} . / \mathrm{kg}$.) after induction with ether. In some animals the trachea was cannulated to ensure a good airway throughout the experiment. The skull was exposed through a longitudinal incision in the scalp. Ear-plugs of suitable size were chosen and pushed firmly into the external auditory meatuses. The head was then mounted in the stereotaxic apparatus by means of the plugs and adjusted so that the sagittal suture coincided as closely as possible with the mid-line of the instrument. The inferior orbital margins were then secured by means of the lugs (M, M and $N, d N$, Fig. 1). With the aid of a dental burr, holes were drilled in the skull in the positions required for the insertion of electrodes. In order to allow adequate inspection of the eyes, lateral canthotomies were performed and the upper eye-lids elevated slightly with sutures.

The intra-ocular pressures were recorded photographically using manometers connected directly to needles inserted in the eyes (Greaves and Perkins, 1952). The systemic blood pressure was similarly recorded from a cannula in the femoral artery. The movements of the nictitating membranes were recorded on the same film by means of threads running from the membranes (Fig. 2, overleaf) over pulleys (F, F, Fig. 1a) to levers on which small mirrors were mounted.

\section{Stimulation}

(a) Electrode.-Unipolar stimulation was used throughout, the cathode being in the brain, and the anode, a brass rod, in the rectum. The electrode itself was a $0.1-\mathrm{mm}$. diameter nickel wire inside a glass capillary tube of $0.125-\mathrm{mm}$. bore and $0.8-\mathrm{mm}$. external diameter. This type of electrode was chosen on account of its rigidity, reliability, and simplicity.

(b) Stimulus.-Square wave, direct current pulses of $1 \mathrm{msec}$. duration at a frequency of $30 \mathrm{c} / \mathrm{s}$ were provided by an electronic generator (Attree, 1950). These values were found suitable in a preliminary series of experiments in which maximal pupillary dilatation was obtained with the electrode in the lateral hypothalamus. With a resistance of $33,000 \mathrm{ohms}$ in series with the electrode and the output of the generator set at 12 volts, measurements with an oscilloscope in several experiments showed that the current passing during stimulation was $0.2 \mathrm{ma}$. at a potential of 2.8 volts across the electrodes. The circuit and characteristics of the stimulus were unchanged throughout the series of experiments.

(c) Introduction of Electrode.-The electrode was lowered into the brain so that its tip lay $15 \mathrm{~mm}$. above the "zero horizontal" plane, i.e. at $\mathrm{H}+15.3$ minutes later the stimulus was applied for 60 seconds. Subsequent stimulations, each of 


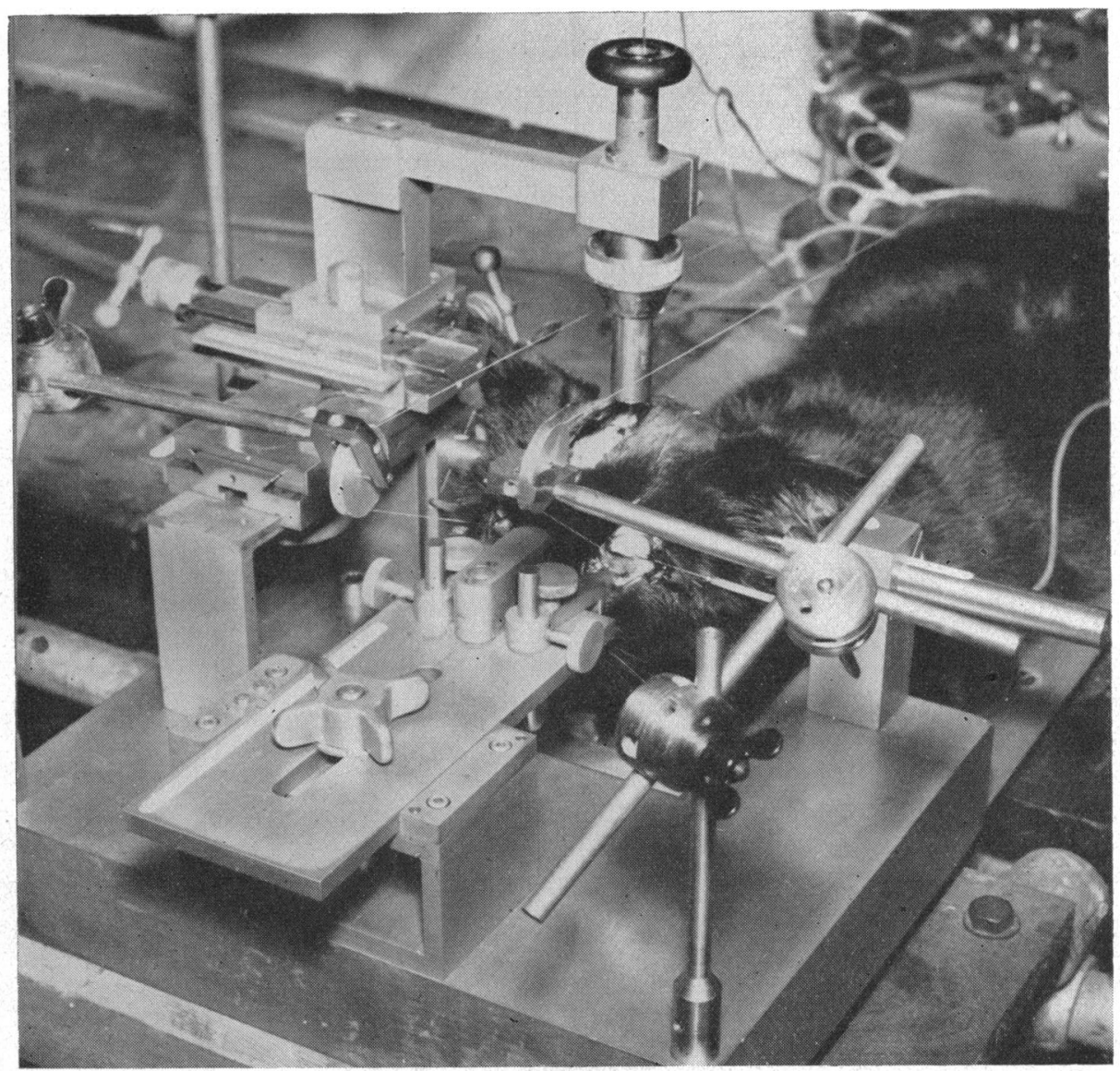

FIG. 2.-The apparatus assembled.

60 seconds' duration, were made at intervals of 5 minutes, the electrode being lowered $1 \mathrm{~mm}$. between stimulations, the lowest position of the tip varying between $\mathrm{H}+3$ and $\mathrm{H}+7$ according to the frontal plane. The electrode was passed down two such tracks on each animal, one on each side of the midline, and generally separated by $7 \mathrm{~mm}$. Only one stimulation was given for each position of the electrode tip.

During each stimulation, the changes in pupillary size were noted together with any ocular movement or muscular contraction in the region of the orbit.

Histology.-When stimulation had been completed, the electrode was withdrawn and six steel marker-needles inserted $7 \mathrm{~mm}$. lateral to the mid-line in Plane 1, and $5 \mathrm{~mm}$. in front of and $5 \mathrm{~mm}$. behind the plane of the electrode tracks. After insertion of the marker-needles, the heart was excised and the head of the cat was elevated for a short time to exsanguinate the cerebral tissues. The brain was then removed and placed in formol-saline. After it had been embedded in paraffin, $10 \mu$ sections were cut and stained with haematoxylin and eosin. Sufficient sections 
were cut to ensure that the lowest point reached by the electrode tip could be recognized.

Assessment of Results.-The responses to each stimulation were entered on specially prepared tables, changes in blood pressure and intra-ocular pressure being recorded quantitatively. The magnitude of the change was judged only from that which occurred during stimulation. Movements of the nictitating membranes were considered to be of significance only when greater than $1 \mathrm{~mm}$. on the tracing. Changes in pupillary size were recorded in the same table, together with observations on any contraction of facial musculature.

The results were then plotted on maps of frontal sections of the diencephalon, the maps being adapted from the atlas of Jasper and Ajmone-Marsan (1955). A separate map in each frontal plane was prepared for each of the following: pupillary reactions, blood pressure changes, nictitating membrane responses, and the correlation between changes in intra-ocular pressure and blood pressure. For convenience, the responses were plotted on one-half only of each map, irrespective of whether the electrode track lay to the right or left of the mid-line.

The positions of points within the brain from which the responses were obtained were mapped only after examination of the slides showing the electrode tracks, allowance being made for shrinkage of the brain-tissue during histological preparation.

\section{Results}

The results will now be presented under four headings:

(1) Pupillary responses.

(2) Nictitating membrane effects.

(3) Blood pressure changes.

(4) Intra-ocular pressure changes.

(1) Pupillary Responses.-Figs $3 a, 3 b$, and $3 c$ (overleaf) show diagrammatically the points from which pupillary changes were elicited in frontal planes $\mathrm{A} 9, \mathrm{~A} 12$, and A15.

Only the responses of the pupil on the same side as the stimulating electrode are shown and, for the sake of simplicity, these ipsilateral responses have been plotted on one-half only of the maps, irrespective of whether the electrode was actually to the right or to the left of the mid-line. This seemed reasonable in view of the fact that the pupillary responses of the ipsilateral and contralateral eyes did not differ significantly in either magnitude or rate of response. It will be seen that, whilst these responses were obtained from widely scattered points in the diencephalon, the most marked effects were encountered in the posterior part of the hypothalamus. Only maps of planes A9, A12, and A15 are shown. Whereas the distribution of responses in planes A10 and A11 did not differ materially from those in $\mathrm{A} 9$ and $\mathrm{A} 12$, there were fewer points yielding pupillary dilatation in the more anterior regions of the diencephalon, viz. in planes A13 and A14. 
The rate of response appeared to be dependent upon the position of the electrode tip. In the hypothalamus the response was rapid, dilatation commencing almost as soon as the stimulus was applied and reaching a maximum 10-15 seconds later; sometimes this maximum was maintained until the end of stimulation, but on other occasions it lasted for only about 30 seconds, after which a gradual decrease in pupillary size was observed during the remaining 20 seconds of stimulation. In the thalamus the pupillary responses were infrequent and small, and the average rate of response often appeared to be slower than that usually seen with hypothalamic stimulation.

Contractions of the pupil were seldom noted, but this was not surprising since under the conditions of our experiment the pupils were normally slits. The only points from which stimulation produced pupillary constriction were in the optic tract in plane $\mathrm{A} 12$, and from isolated points in A8. This last plane has not yet been examined by us in detail.

A second series of maps was prepared from the results of experiments in which the
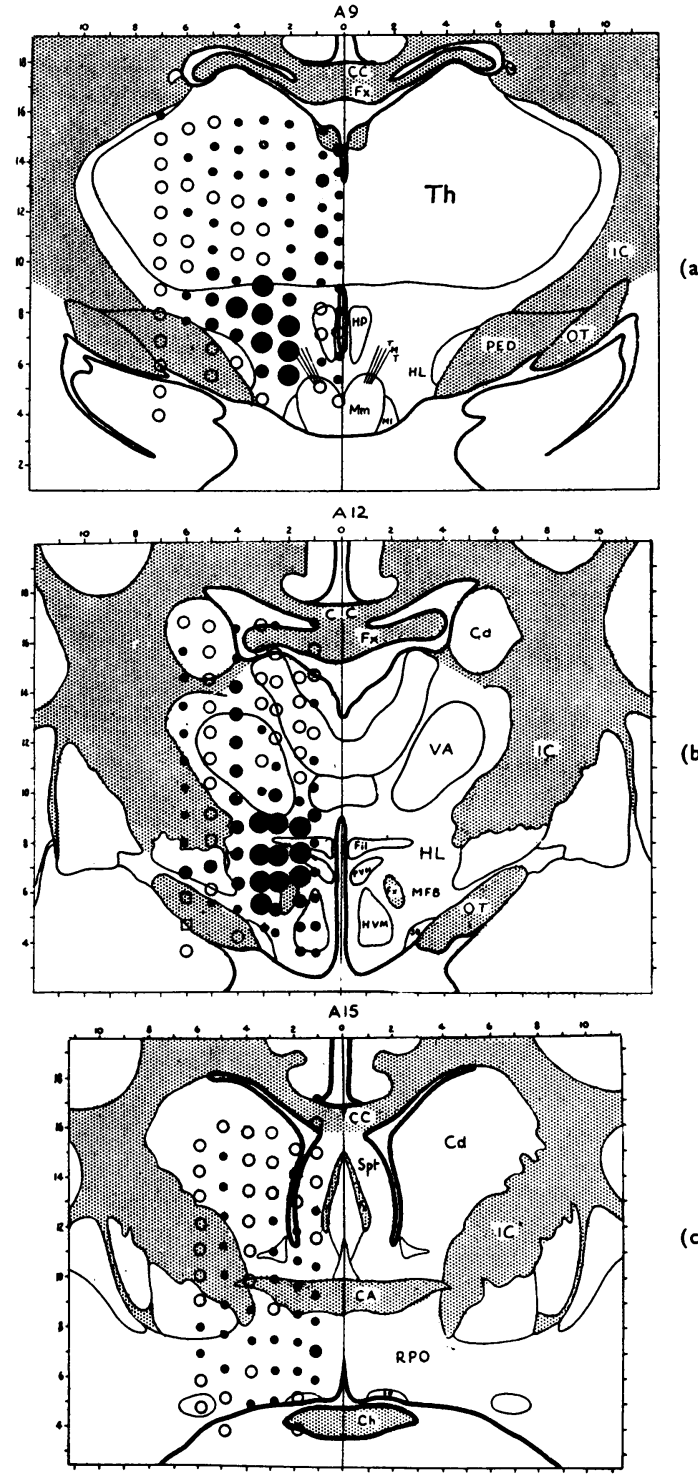

PUPL RESPONSES

- Pupil dilototion kss than $3 \mathrm{~mm}$. 口 Pupil constrietion.

- Pupil dilotation 3-6mm. - No effect.

(b)

FIG. $3(a),(b),(c)$.-Pupillary responses in frontal planes A9, A12, and A15. For explanation of lettered symbols see Appendix.

positions of stimulation had been found histologically to duplicate those used in the first series. Although the second group of maps is incomplete, there is considerable agreement between the two series. 
(2) Nictitating Membrane Effects.-Nictitating membrane responses were not commonly observed (68 responses in 757 stimulations), and when elicited were small compared with the effect obtained by stimulation of the cervical sympathetic trunk. Both contractions and relaxations were recorded, but the latter were extremely small and less frequent. Ipsilateral effects occurred more frequently than contralateral effects.

With regard to contractions, we noted that the nictitating membrane tracing exhibited two types of movement:

(1) a fairly rapid rise followed by a slow return (e.g. as on Fig. 10);

(2) an abrupt rise at onset and an equally abrupt fall at the cessation of stimulation (e.g. as on Fig. 9).

After further examination of these tracings, we concluded that the former reflected smooth muscle activity whilst the latter suggested that striated muscle was in some way involved, possibly through attachments between the recti muscles and orbital smooth muscle.

The first type of effect was never accompanied by movement of the eyes and was sometimes bilateral, although the ipsilateral response was generally greater. This type of response was obtained from the hypothalamus in the frontal planes A11 and A12, mostly in the region of the anterior column of the fornix.

The second type of effect was always ipsilateral and was sometimes accompanied by movement of the eye on the same side. On one occasion, histological examination revealed that the electrode had passed through the ventral surface of the hypothalamus, the tip presumably being in the neighbourhood of the third cranial nerve. All other responses of this type were obtained when the electrode tip was in the extreme ventral part of the hypothalamus about $2-3 \mathrm{~mm}$. from the mid-line.

(3) Blood Pressure Responses.-Since the intra-ocular pressure is known to be influenced by changes in systemic blood pressure, this latter was recorded continuously. A brief account will now be given of the changes in the blood pressure per se.

Simple rises and falls, and complex responses of the blood pressure were obtained from a wide area of the diencephalon, but, in contrast to the pupillary effects, these responses showed considerable variation from animal to animal. Thus, stimulation of a particular area within the diencephalon might be accompanied by a rise in blood pressure in one animal, whilst stimulation of the same area in another might result in a fall. There was some indication from the results that the type of response was partly dependent upon the general level of the blood pressure; in animals with a high initial blood pressure stimulation was frequently accompanied by a fall in blood pressure and vice versa.

The response usually started promptly at the commencement of stimulation 
and sometimes persisted for a period after the stimulus was switched off or was succeeded by a temporary change in the opposite direction.
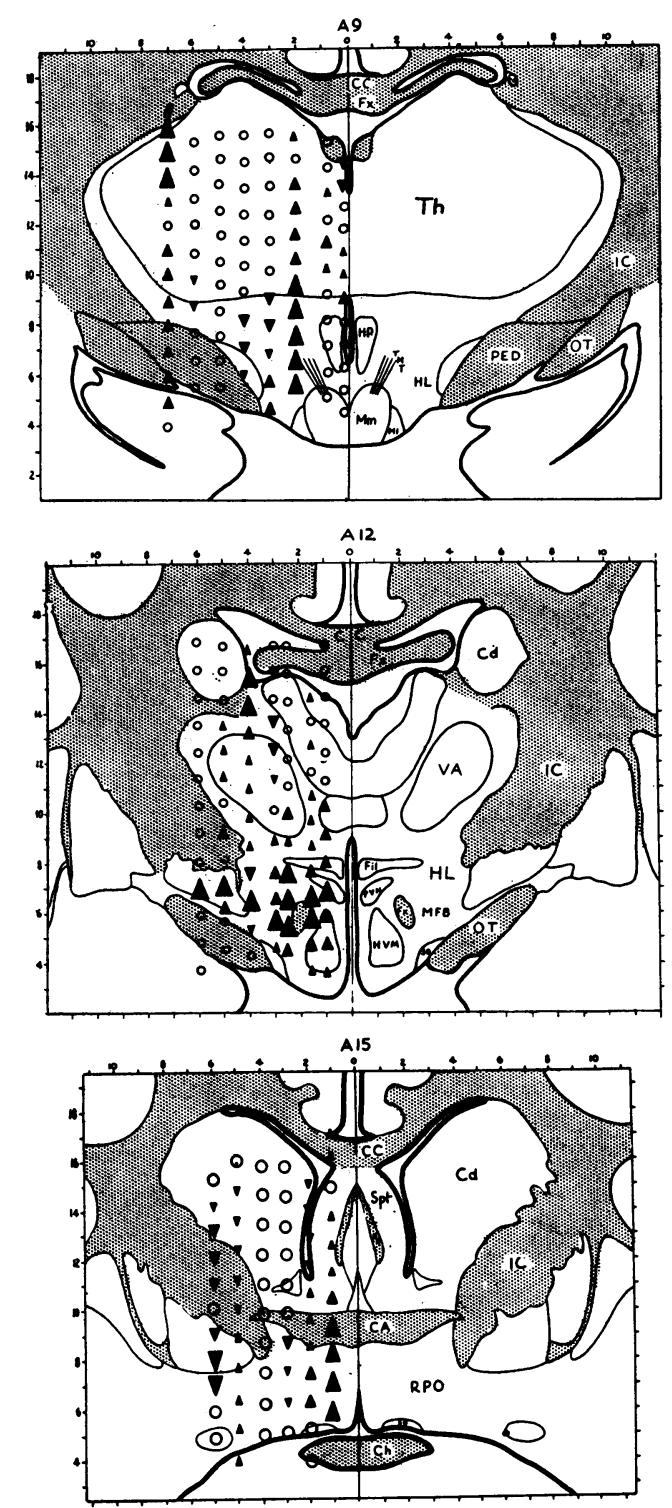

BLOOD PRESSURE RESPONSES

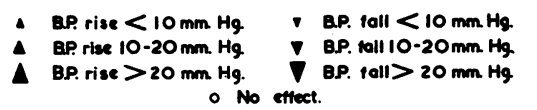

FIG. $4(a),(b),(c)$.-Blood pressure responses in frontal planes A9, A12, and A15. For explanation of lettered symbols see Appendix.
With regard to localization, the maps (Figs $4 a, 4 b$, and $4 c$ ) show that most responses were obtained from the hypothalamus, rises in blood pressure predominating in its medial part; this (a) was noticeably so in planes A12 and A13. In the lateral hypothalamus, falls in blood pressure were not uncommon. Responses were also obtained from the thalamus, but these were relatively small.

\section{(4) Intra-Ocular Pressure Effects}

(a) Types of Response.-In the previous section we drew attention to the influence of the systemic blood pressure on intra-ocular pressure. It is of considerable importance therefore to take into account all such changes when analysing intra-ocular pressure responses, and the simultaneous recording of these effects allows this essential comparison to be made. For the purpose of this analysis, we have divided the responses into nine groups; the Table shows the incidence of the responses obtained in each group for both ipsilateral and contralateral eyes out of a total series of 745 stimulations. This Table has been compiled from two series of experiments, the second of which consists of duplications 
of positions in the first series and is incomplete. The inclusion of the incomplete series does not alter materially the distribution of responses over the nine groups, since an analysis of the first complete series agreed with that presented for the combined results.

TABLE

PERCENTAGE INCIDENCE OF VARIOUS COMBINATIONS OF RESPONSES OF INTRA-OCULAR PRESSURE AND BLOOD PRESSURE IN 745 STIMULATIONS

Number of responses in each group is given in brackets

\begin{tabular}{c|c|c|c|c|c|c}
\hline \multirow{2}{*}{$\begin{array}{c}\text { Blood } \\
\text { Pressure }\end{array}$} & \multicolumn{5}{|c}{ Intra-Ocular Pressure } \\
\cline { 2 - 7 } & Rise & No Change & Fall & Rise & No Change & Fall \\
\hline Rise & $17.0(127)$ & $17.4(130)$ & $2.6(19)$ & $16.5(123)$ & $19.5(145)$ & $1.2(9)$ \\
\hline No Change & $7.9(59)$ & $44.4(331)$ & $0(0)$ & $6.0(45)$ & $46.0(343)$ & $0 \cdot 1(1)$ \\
\hline Fall & $3.5(26)$ & $6.2(46)$ & $0.9(7)$ & $3.2(24)$ & $6.3(47)$ & $1.1(8)$ \\
\hline
\end{tabular}

In general, each type of response occurs with the same frequency in the two eyes. The occasions on which both intra-ocular pressure and blood pressure showed no change comprise almost half the total number of stimulations. Slightly more than half of the number of stimulations which were accompanied by intra-ocular pressure changes were also accompanied by changes in blood pressure in the same direction. The most interesting intra-ocular pressure changes were those which were independent of blood pressure changes, and these occurred in approximately 15 per cent. of stimulations in the ipsilateral eye and in 10 per cent. in the contralateral eye, an independent rise being much more common than a fall.

Figs 5 to 11 (overleaf) constitute a series of tracings illustrating the various effects already described.

(b) Localization of Points from which Independent Changes in Intra-Ocular Pressure were Obtained.-In Figs 12(a)-12(g) the responses of the intra-ocular pressure which were independent of blood pressure changes are correlated with the points stimulated in the diencephalon for frontal planes A9 to A15 inclusive. Only the responses of the ipsilateral eye are shown. The effects in the contralateral eye, though similar, were less numerous, but the distribution of points from which they were obtained was essentially the same as for the ipsilateral eye.

It should be noted that independent falls in intra-ocular pressure resulted from stimulation in the immediate vicinity of the anterior column of the fornix, whilst independent rises were obtained from a much wider area of the diencephalon. 


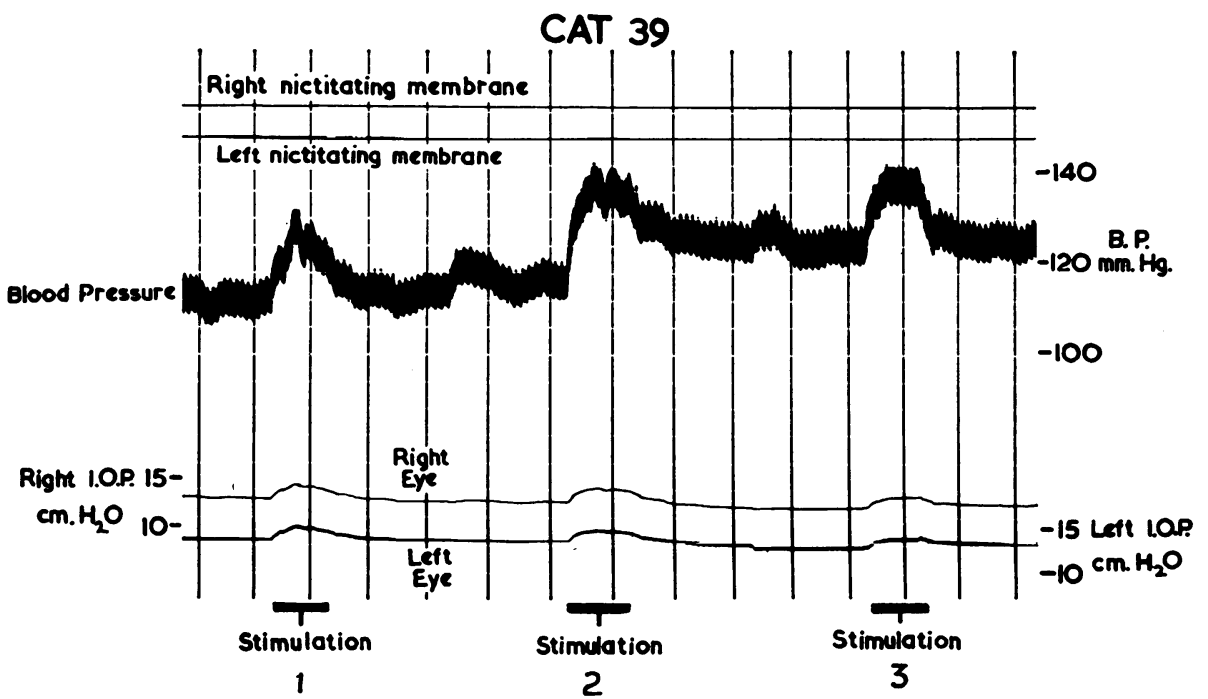

Fig. 5.-Tracing showing rises in intra-ocular pressure combined with blood pressure changes in the same direction. Interval between vertical lines represents $60 \mathrm{sec}$.

Localization: A14, R2 (see Fig. 12f)

Stimulation 1: $\mathrm{H}+7$

Stimulation 2: $\mathrm{H}+6$

Stimulation 3: $\mathbf{H}+5$

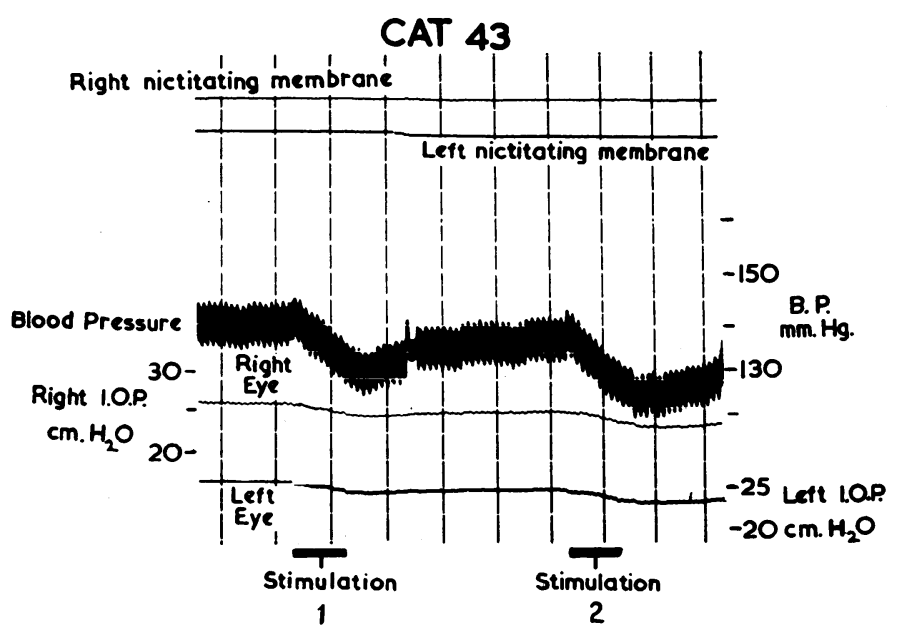

Fig. 6.-Tracing showing falls in intra-ocular pressure combined with falls in blood pressure. Interval between vertical lines represents 60 sec.

Localization: A15, R2 (see Fig. 12g)

Stimulation 1: $\mathrm{H}+14$

Stimulation 2: $\mathrm{H}+13$ 


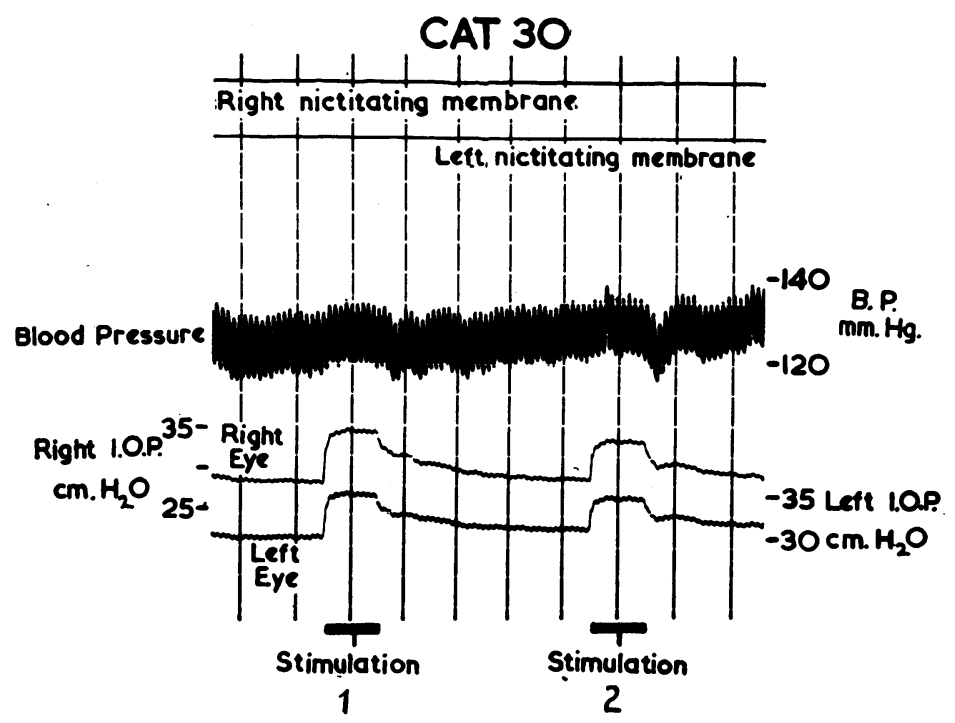

FIG. 7.-Tracing showing marked independent rises in intra-ocular pressure without blood pressure responses. Note abrupt rise and fall in intra-ocular pressure response. Interval between vertical lines represents $60 \mathrm{sec}$.

Localization: A9, R3 (see Fig. 12a)

Stimulation 1: $\mathrm{H}+12$

Stimulation 2: $\mathrm{H}+11$

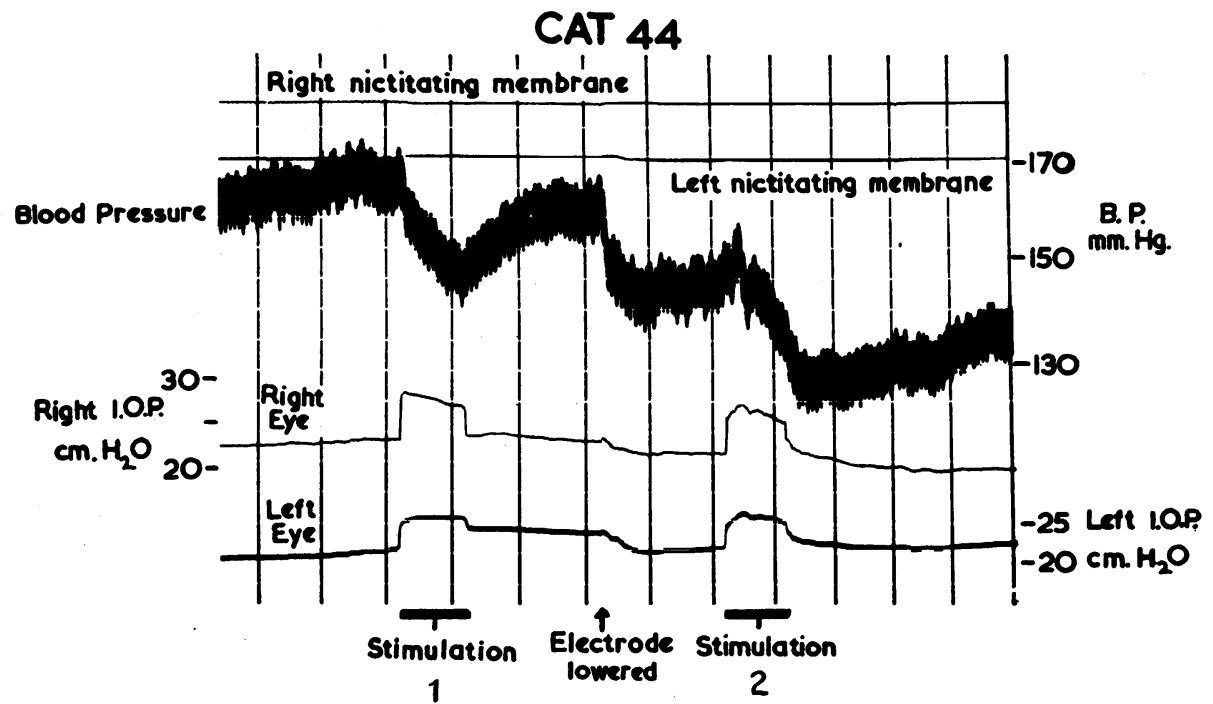

FIG. 8.-Tracing showing a rise in intra-ocular pressure occurring simultaneously with a fall in blood pressure. Again the intra-ocular pressure change is abrupt. Lowering the electrode has resulted in a fall in blood pressure and a fall in intra-ocular pressure. Interval between vertical lines represents $60 \mathrm{sec}$.

Localization: A9, L4 (see Fig. 12a)

Stimulation 1: $\mathrm{H}+8$

Stimulation 2: $\mathrm{H}+7$ 


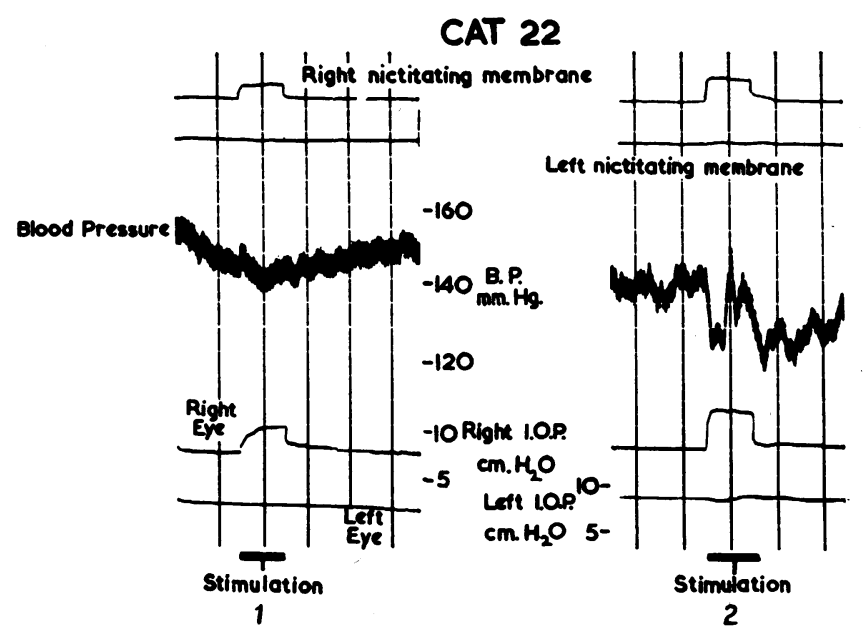

FIG. 9.-Tracings showing an independent rise in intra-ocular pressure accompanied by a contraction of the ipsilateral nictitating membrane. The abrupt movement of the latter suggests striated muscle activity. See text. Interval between vertical lines represents $60 \mathrm{sec}$.

Localization: A11, R4 (see Fig. 12c)

Stimulation 1: $\mathrm{H}+6 \frac{1}{2}$

Stimulation 2: $\mathrm{H}+5 \frac{1}{2}$

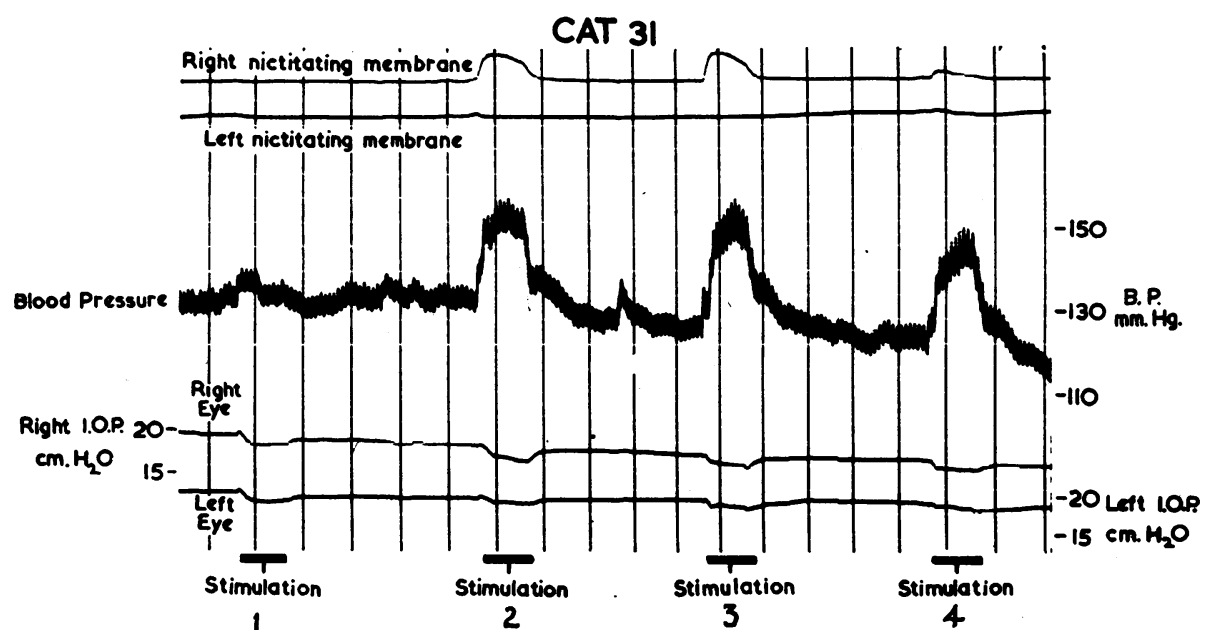

FIG. 10.-Tracing showing falls in intra-ocular pressure with rises in blood pressure. Note marked rises in blood pressure and contractions of right nictitating membrane in Stimulations 2-4. Interval between vertical lines represents $60 \mathrm{sec}$.

Localization: A12, R1.5 (see Fig. 12d)

Stimulation 1: $\mathrm{H}+8 \frac{1}{2} \quad$ Medial hypothalamus

Stimulation 2: $\mathbf{H}+7 \frac{1}{2}$ Approximately level of fornix

Stimulation 3: $\mathrm{H}+6 \frac{1}{2}$

Stimulation $4: \mathrm{H}+5 \frac{1}{2}$ 


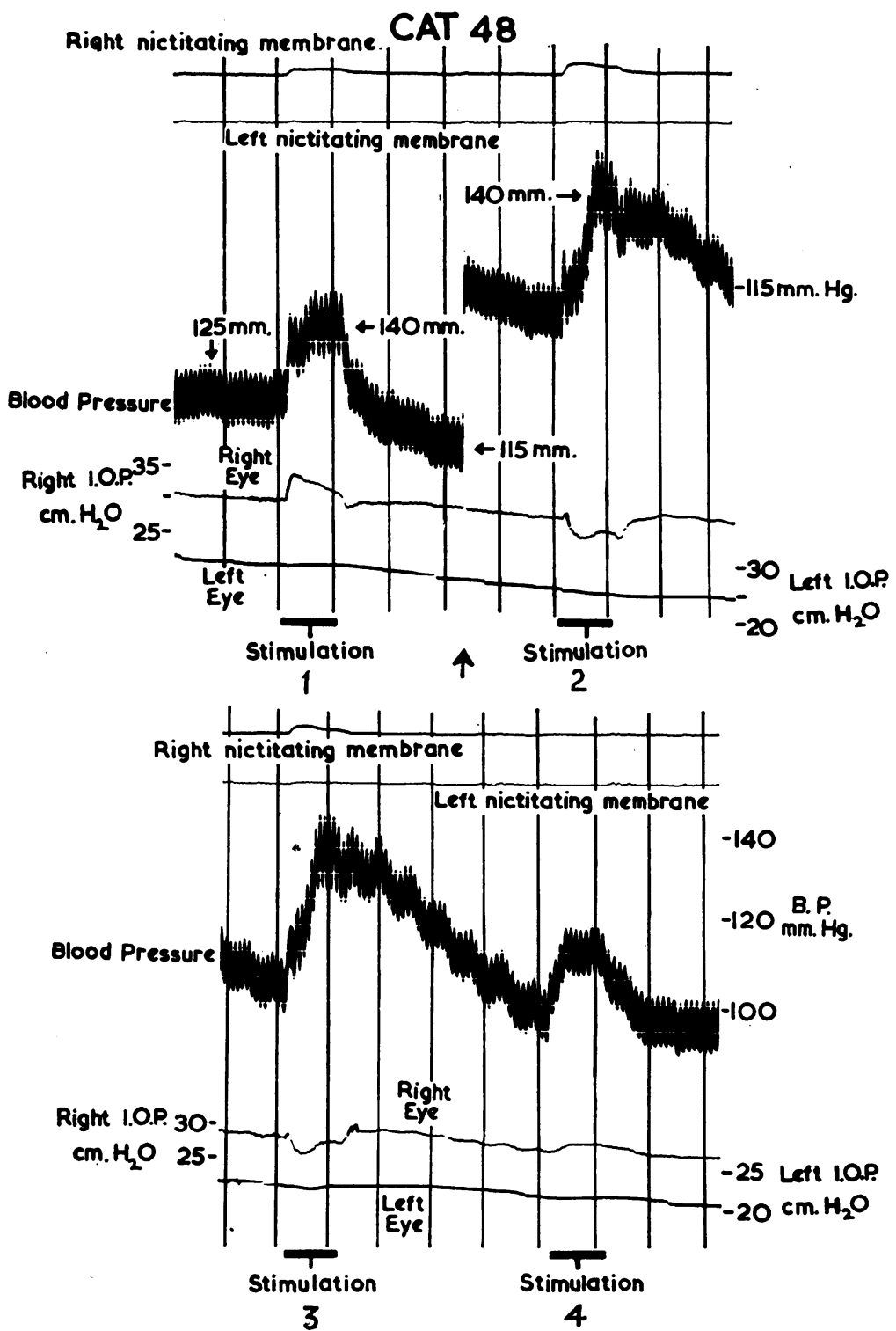

FIG. 11.-Tracings showing fall in intra-ocular pressure with a rise in blood pressure obtained from a limited area. The points stimulated are $1 \mathrm{~mm}$. apart and only Stimulations 2 and 3 give the characteristic response. Interval between vertical lines represents $60 \mathrm{sec}$.

Localization: A11, R2 (see Fig. 12c)

Stimulation 1: $\mathrm{H}+71$

Stimulation 2: $\mathrm{H}+6 \frac{1}{4}$

Stimulation 3: $\mathrm{H}+5$

Stimulation $4: \mathrm{H}+4$

( $\uparrow=$ B.P. tracing adjusted) 

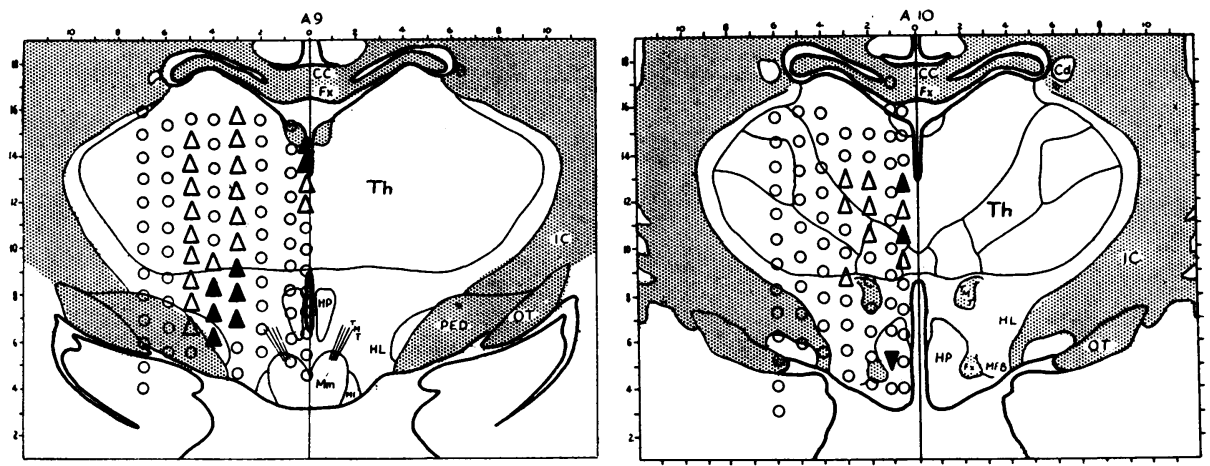

(a)

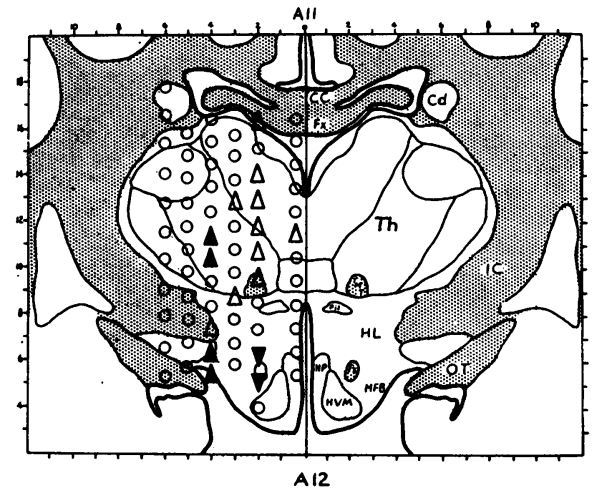

(b)

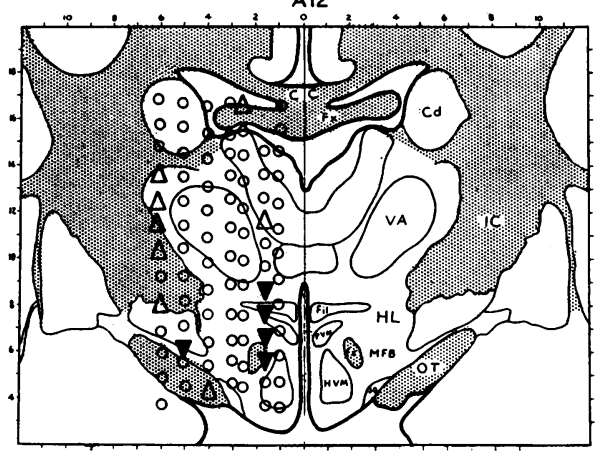

(c)

INTRA-OCULAR PRESSURE RESPONSES

$\triangle$ I.QP. rise with no change of B.P.

$\Delta$ l.ap rise with QR. roll.

$\nabla$ LO.P tall with no chonge of BP.

$\nabla$ I.O.P. foll with B.P rise.

O Other types of response

Fig. $12(a-g)$. - Changes in intra-ocular pressure occurring independently of blood pressure changes in frontal planes A9 to A15. For explanation of lettered symbols see Appendix.

\section{Discussion}

The foregoing results have shown that electrical stimulation of the diencephalon causes changes in intra-ocular pressure, and many of these 
(e)

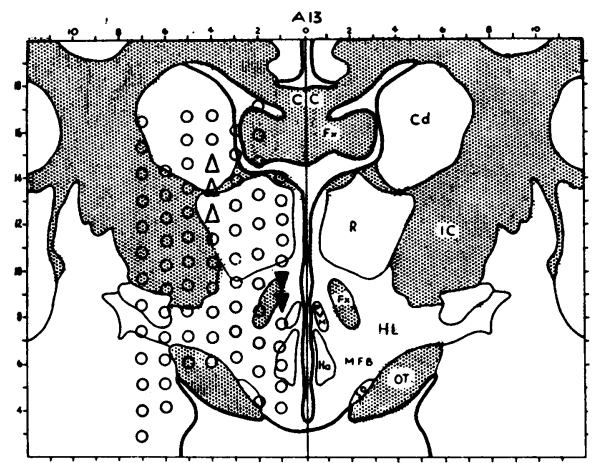

(f)

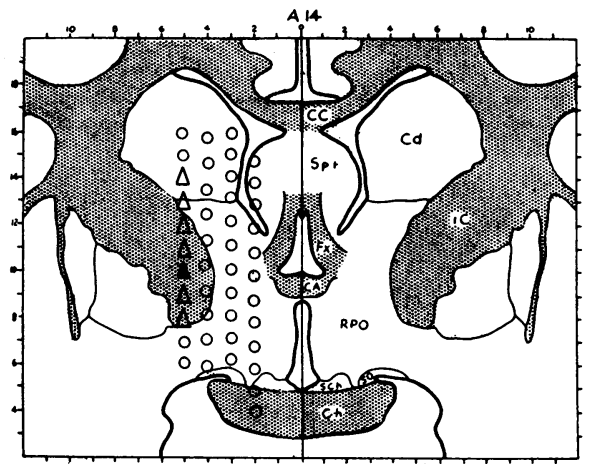

(g)

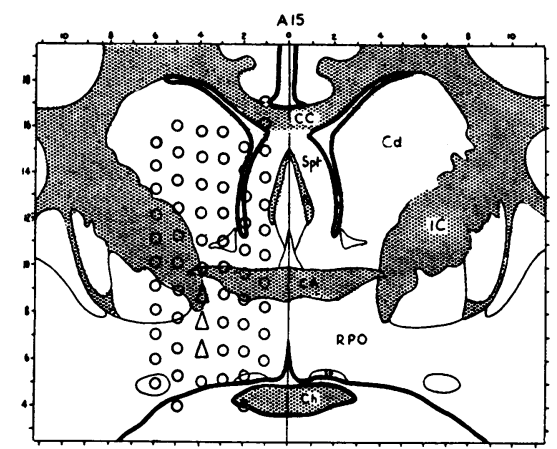

changes reflect similar trends in the systemic blood pressure. This is broadly in agreement with the findings of von Sallmann and Lowenstein (1955), and we have encountered as wide a variety of type and pattern of response as did these authors. However, a strict comparison is hardly permissible for the following reasons:

(1) The stimulus employed by these authors differed in certain characteristics from that used by us;

(2) These authors examined the area in a different way, concentrating on a parasagittal plane $2 \mathrm{~mm}$. from the mid-line as compared with our wider coverage of the diencephalon;

(3) These authors used the speed of change in the intraocular pressure as a criterion in classifying their responses, whereas our analysis is based primarily on the correlation of such changes with changes in blood pressure.

This has enabled us to recognize certain well-defined independent responses of which the most interesting is a fall in intra-ocular pressure accompanying a rise in blood pressure.

It was hoped that the subsidiary observations on the pupils, nictitating membranes, and blood pressure would be helpful in the interpretation of the responses of the intra-ocular pressure, and these observations will now be discussed. With regard to changes in the pupil, it was pointed out earlier that our method favoured the observation of pupillary dilatation, the pupils being usually small when the experiment had reached the stage at which the stimulations were commenced. Thus, whilst the slightest pupillary dilatation was readily seen, a corresponding degree of constriction would have passed unnoticed. In general, dilatation was elicited from points over a wide area, but the greatest responses were obtained from the posterior 
half of the hypothalamus. This is in agreement with the findings of Ranson and Magoun (1933). The occurrence of pupillary dilatation cannot be taken as evidence of sympathetic activity, for Weinstein and Bender (1941) and Carlson, Darrow, and Gellorn (1940) have shown that in the cat pupillary dilatation resulting from diencephalic stimulation is not abolished by section of the cervical sympathetic trunk, and that, in all probability, the response is partly due to inhibition of the parasympathetic outflow in the third cranial nerve. We have confirmed these observations and our experiments will be reported later. Retraction of the nictitating membrane appears to be a more reliable indicator of sympathetic activity, although, as was pointed out earlier, it is necessary to distinguish the true smooth muscle response from that due to striated muscle connected to the orbital smooth muscle.

Whereas pupillary dilatations were elicited mainly from the posterior half of the hypothalamus, the points yielding elevation of blood pressure were not limited to this region but were in fact widely scattered throughout the diencephalon. No consistent relationship was found between the type of variation in the blood pressure and the region stimulated, both rises and falls sometimes being obtained from neighbouring points. A review of our results strongly suggested that the type of response was to a certain extent determined by the reactivity of the particular animal and the level of its blood pressure. This lack of consistency, as regards both the type of response and its localization, suggests that the underlying mechanism of the response of blood pressure is complex. The usefulness of these observations in explaining the mechanism of accompanying changes in intra-ocular pressure is therefore limited, but there is still a need to correlate these responses of intra-ocular pressure and blood pressure, since the changes in intra-ocular pressure which are of greatest interest are those which do not follow changes in blood pressure.

These latter responses fall into two groups:

(1) those in which the intra-ocular pressure rises independently of blood pressure;

(2) those in which a fall in intra-ocular pressure occurs without an accompanying fall in blood pressure.

The abrupt nature of the independent elevation in intra-ocular pressure suggests that this may have been due to contraction of striated or nonstriated muscle within the orbit, although a vascular cause cannot as yet be excluded. The subsidiary observations on blood pressure, nictitating membrane, and pupillary changes do not provide any clue to the mechanism. With regard to the independent fall in intra-ocular pressure, it was thought that this might be due to a fall in capillary blood pressure within the eye as a result of vasoconstriction brought about by increased sympathetic activity. This view is supported by the subsidiary observations, the fall in intra-ocular pressure being accompanied by a rise in systemic blood pressure, pupillary 
dilatation, and sometimes by contraction of the nictitating membrane. This response has been investigated further and the findings will be reported subsequently.

In considering the localization of points yielding changes in intra-ocular pressure, it has already been pointed out that the blood pressure responses showed no pattern of localization, and therefore the same is true for such intra-ocular pressure responses as may occur as reflections of changes in blood pressure. With regard to independent rises in intra-ocular pressure, these were elicited from a fairly wide area, but in the main were limited to the thalamus; several points giving this response were found within the internal capsule and in the posterior hypothalamus in plane A9. On the other hand, the independent falls in intra-ocular pressure were confined, with the exception of one point in A12, to the medial part of the hypothalamus, from plane A10 to plane A13, and most of these points were closely related to the anterior column of the fornix. von Sallmann and Lowenstein (1955) also obtained independent rises in intra-ocular pressure from a wide area, predominantly from the thalamus, and our findings regarding the localization of this type of response are therefore in general agreement. However, while these authors reported independent falls in intra-ocular pressure from both the hypothalamus and thalamus, we obtained this response from the hypothalamus only, and never from the thalamus.

In view of our present ignorance of the mechanism of the independent rises in intra-ocular pressure and the wide area from which they were elicited, it is difficult to assess their significance. Combined rises in intra-ocular pressure and blood pressure were obtained from stimulation of many points throughout the hypothalamus, as reported by von Sallmann and Lowenstein (1955), who suggested that these effects were manifestations of a mass sympathetic discharge. If this is the case, then the independent fall in intra-ocular pressure which we obtained from a more circumscribed area in the hypothalamus may possess additional significance; we have already suggested that it may be due to a reduction in capillary blood pressure within the eye, and, in the presence of a rise in systemic blood pressure, this could be due only to a local vasoconstriction resulting from local sympathetic activity outweighing the effects of the general sympathetic discharge. The present evidence does not allow the conclusion that the distribution of this "local" sympathetic activity is confined to the ocular tissues.

The responses in which the changes in intra-ocular pressure merely reflect changes in blood pressure must be considered as non-specific, similar responses being obtained, for example, after a painful stimulus or in acute anoxia. On the other hand, the responses of intra-ocular pressure which occur independently of blood pressure are more specific and provide the only indication that the diencephalon might have a special role in the maintenance of the intra-ocular pressure. It is not certain, however, that 34 
the pathways involved in these responses originate in the diencephalon, since it is possible that the effects were obtained from stimulation of nerve tracts running through the diencephalon and emanating from other nervous centres. Nevertheless, our findings are compatible with the view that the diencephalon influences the intra-ocular pressure, but they do not allow the conclusion that any such influence is in the nature of a controlling mechanism. Such a mechanism demands the existence of an afferent pathway from the eye to the diencephalon in addition to the efferent pathways. These latter are, in fact, the only parts of the mechanism which can be demonstrated by stimulation in the central nervous system.

Turning from these purely physiological considerations, it is not possible at present to see how far these findings are applicable to the aetiology of glaucoma, but it should be borne in mind in this connexion that they may have significance irrespective of the existence of any diencephalic control of intra-ocular pressure, as, for example, in explaining the precipitation of an acute rise in ocular tension. It is also reasonable to take into account, not only the centrally induced changes in intra-ocular pressure, but also the possible alterations in the vascular supply to the eye, which may be the more important feature in some forms of glaucoma by giving rise to defects in the visual fields as a result of relative ischaemia of the neuro-retinal elements.

We would emphasize, however, that these remarks are speculative and that no firm clinical inferences can be drawn at present, but the experimental finding that stimulation in the diencephalon does influence intra-ocular pressure undoubtedly encourages further investigation.

\section{Summary}

(1) The diencephalon of anaesthetized cats has been stimulated electrically. The intra-ocular pressure, systemic blood pressure, and movements of the nictitating membranes were recorded. Pupillary responses were also observed. The position of the tip of the electrode was confirmed histologically and the points from which responses were obtained were plotted on maps of the diencephalon.

(2) Pupillary dilatations were elicited from a wide area of the diencephalon, the greatest effects being obtained from stimulation in the hypothalamus in frontal planes 9 to $13 \mathrm{~mm}$. anterior to the inter-aural line (A9 to A13). Dilatations were usually of equal extent in the two eyes. Pupillary constrictions were rarely found.

(3) Responses of the nictitating membranes were infrequent and small in magnitude, the most common being a small retraction.

(4) Simple rises and falls with some complex responses of the blood pressure were obtained from a wide area, but the type of response differed considerably from animal to animal. The most marked rises in blood 
pressure occurred with stimulations in the medial hypothalamus in frontal planes A12 and A13.

(5) The responses of intra-ocular pressure were analysed with reference to the accompanying changes in blood pressure. In almost half the total number of stimulations, both intra-ocular pressure and blood pressure remained unchanged. In many instances in which responses were obtained, the changes in intra-ocular pressure were accompanied by changes in blood pressure in the same direction.

(6) Independent rises in intra-ocular pressure were observed, the blood pressure either falling simultaneously or remaining unchanged. These responses were obtained from widely separated points, mainly in the posterodorsal region of the diencephalon.

(7) Independent falls in intra-ocular pressure formed part of a characteristic response-pattern in which there was an accompanying rise in blood pressure, pupillary dilatation, and sometimes retraction of the nictitating membrane. This was obtained from a fairly circumscribed area in the medial hypothalamus. It is suggested that the fall in intra-ocular pressure in this type of response is due to a reduction in the ocular blood supply.

(8) These findings are discussed with regard to the ways in which the intra-ocular pressure may be influenced by central nervous mechanisms.

We are grateful to Sir Stewart Duke-Elder for the advice and encouragement he has given us in this investigation. We should also like to acknowledge with thanks a grant from the Alexander Pigott Wernher Research Fund.

\section{REFERENCES}

Attree, V. H. (1950). J. sci. Instrum., 27, 43.

Carlson, H. B., Darrow, C. W., and Gellhorn, E. (1940). Amer. J. Physiol., 129, P329.

DukE-Elder, S. (1957). Trans. ophthal. Soc. U.K., 77, (in press).

Gloster, J., and Greaves, D. P. (1956). Proc. roy. Soc. Med., 49, 675.

Greaves, D. P., and Perkins, E. S. (1952). British Journal of Ophthalmology, 36, 258.

- (1953). Ibid., 37, 54.

JASPER, H. H., and AJMONE-MARSAN, C. (1955). "Stereotaxic Atlas of the Diencephalon of the Cat." Nat. Res. Counc., Ottawa, Canada.

NagaI, M., Ban, T., and Kurorsu, T. (1951). Med. J. Osaka Univ., 2, 433.

Perkins, E. S. (1955). " “XVII Concilium Ophthal. 1954, Canada, U.S.A., Acta," vol. 2, p. 1157. University of Toronto Press.

(1957). British Journal of Ophthalmology, 41, 257.

Ranson, S. W., and Magoun, H. W. (1933). Arch. Neurol. Psychiat. (Chicago), 29, 1179.

SAllmanN, L. Von, and Lowenstein, O. (1955). Ibid., 39, No. 4 Pt. 2, p. 11.

Schmerl, E., and Steinberg, B. (1950). Amer. J. Ophthal., 33, 1379.

Weinstein, E. A., and Bender, M. B. (1941). J. Neurophysiol, 4, 44.

Weinstein, P. (1954). Ophthalmologica (Basel), 127, 164.

\section{APPENDIX}

List OF LETTERED SYMBols

Anterior commissure

Corpus callosum

Caudate nucleus

Optic chiasma 
Fil Nucleus filiformis

Fx Fornix

$\mathrm{Ha}$ Anterior hypothalamic nucleus

HL Lateral hypothalamic area

HP Posterior hypothalamic nucleus

HVM Ventromedial hypothalamic nucleus

IC Internal capsule

MFB Medial forebrain bundle

MI Lateral mamillary nucleus

Mm Mamillary body

OT Optic tract

PED Cerebral peduncle

PVH Paraventricular hypothalamic nucleus

$R \quad$ Nucleus reticularis

RPO Pre-optic area

SCh Supra-chiasmatic nucleus

SO Supra-optic nucleus

Spt Area septalis

Th Thalamus

TMT Mamillo-thalamic tract

VA Nucleus ventralis anterior of thalamus 